\title{
Concurrent Multi-Modality Treatment of Keloids (CMTK) Not Manageable by Conventional Postoperative Radiotherapy
}

\author{
Kamal Malaker $^{{ }^{*}}$, Mustafa Zaidi ${ }^{2}$, Mohamad Rida Franka ${ }^{2}$, Tawfik Al Yafi ${ }^{3}$ \\ ${ }^{1}$ Introduction to Clinical Medicine, Ross University School of Medicine, Roseau, Dominica; ${ }^{2}$ Burns and Plastic Surgery Centre, \\ Burns and Plastic Surgery Hospital, Tripoli, Libya; ${ }^{3}$ Plastic Surgery, King Khalid National Guard Hospital, Jeddah, KSA. \\ Email: ${ }^{*}$ Kamal_malaker@hotmail.com
}

Received February $21^{\text {st }}, 2013$; revised March $12^{\text {th }}, 2013$; accepted May $2^{\text {nd }}, 2013$

Copyright (c) 2013 Kamal Malaker et al. This is an open access article distributed under the Creative Commons Attribution License, which permits unrestricted use, distribution, and reproduction in any medium, provided the original work is properly cited.

\begin{abstract}
Objective: To design and test a treatment regimen which is clinically responsive, readily available, cost effective, and applicable especially to children and women of child bearing age. Design Setting: A prospective cohort study. Setting: Two major postgraduate teaching hospitals: one in Tripoli, Libya and the other in Jeddah, Saudi Arabia. Participants: Fifty-seven patients with 79 keloids, referred from Plastic Surgery Units between April 1996 and January 2005. Main Outcome Measure: Degree of flattening of the keloidal lesion and symptomatic recovery. Results: Result of treatment has been analyzed using unified set criteria. Seventy-seven percent of this cohort had complete response. $19 \%$ of cases had partial response, 50\% acknowledged the treatment outcome had been "satisfactory" and $44 \%$ had an "acceptable" outcome. There was no significant acute or delayed reaction. Conclusion: The technique appears universally adaptable, cost effective, and can safely be prescribed for children and women of child-bearing age. In spite of prolonged treatment course, compliance was excellent.
\end{abstract}

Keywords: Unresceptable Keloids; Non-Surgical Treatment

\section{Introduction}

Postoperative radiotherapy is generally effective for keloid treatment [1], but in several situations neither surgery nor radiotherapy is applicable. Symptomatic keloids could seriously affect a patient's life. Only sufferers can understand the agony; the itching and irritation is deep and there is no relief by scratching or from any topical applications. Clinical experience suggests that the psychological effect is usually devastating, especially with extensive keloids on exposed areas. This is due to the physical disfigurement and silent rejection by society, even at times by family and friends. To the medical profession, not being a life threatening condition, it is of minimal consequence, and patients are frequently passed from generalist to specialist doctors, in vain.

Although the etiology of keloids remains an enigma, in the last 10 - 15 years significant advances have been made in understanding the natural history of keloids and the biogenetic processes involved [2]. As a result, the

"Corresponding author. current approach to prevention of keloid formation is biological, for example, interference with TNF, PDGF, ILI, cytokine activity [3], Tamoxifen analog that down regulates TGF [4], anti-allergic drug Tronilast that suppresses release of cytokines as PDGF, TGF, and Interlukin-1b [5] are currently under trial. Unfortunately, none of these biological developments are accessible to patients where they are needed most, namely in the developing world. The research however, is not directed against well-informed matured keloids for its resolution.

There is ample clinical evidence to suggest that:

- Intra-lesional infiltration of steroids can alleviate symptoms of keloids, and can even induce atrophy and wound dehiscence [6-8].

- Persistent pressure can induce atrophy of keloidal scars [9-11].

- Silicone sheets and gels cause symptomatic relief and softening of keloids by moisture retention [12-15].

- Clinical application of post-operative radiotherapy for the prevention of keloids is well established [16].

- Carcinogenic effect of radiation in children [17] and 
women (breast) is well documented [10,18]. Radiation induced skeletal growth defect in children is also well recognized [19].

With the above basic understanding we set out to make best use of the above factors in developing a technique to treat large symptomatic keloids, which are not amenable to any form of therapeutic intervention, to date.

\section{Objective}

This study was undertaken to develop a method of treatment for patients with recurrent, symptomatic (physical or psychological) keloids, which could not be treated due to location, size, or extent by surgery and/or radiotherapy especially due to young age and women of childbearing age. The primary objective was to relieve symptoms, be it physical, cosmetic, or psychosocial, using a combination of available treatment modalities.

\subsection{Rationale for Development of the Technique}

Intra-lesional infiltration of a long acting steroid has a well-established place in the treatment of symptomatic keloid and hypertrophic scars [6-8]. Cortisone is known to be a potent anti-angiogenic agent $[14,20,21]$. Heparin has shown to possess strong anti-angiogenic property in association with cortisone [7,11,15,22].

Physical pressure applied on the keloidal lesions, by dint of causing hypoxia, we presume, would induce atrophy of the hypertrophic scar or matured keloid. Thus, it is hoped, if pressure is applied along with intra-lesional long-acting steroid in combination with Heparin, the possibility of inducing atrophy is expected to be substantially increased. It is hoped that the anti-angiogenic property of steroid and heparin would work along with ischemia, induced by physical pressure leading to atrophy and eventual resolution of keloidal tissue.

Silicone sheet dressing apparently causes softening of keloids by retaining moisture [12-14]. It has been suggested that this is induced by static electricity generated by the sheet and skin [15]. Retention of interstitial fluid might improve keloidal oxygen diffusion and reduce hypoxia. Hypoxia is a strong potentiator of tissue angiogenesis $[6,23,24]$ and, at the same time, fibrosis, which is evident in many clinical conditions $[1,6,24,25]$. Softer or oedematous keloids are likely to be more amenable to dissemination of medication i.e., instilled steroids and/or Heparin. Added pressure assists in dissemination of drugs more efficiently than it would through tightly packed bundles of fibrous tissue in keloids, without oedema or softer consistency.

To achieve this, pressure needs to be reasonably high. It must be continuous for 2 - 3 weeks, by which time tissue reconstitution following any injury would have been completed [26]. Unfortunately, constant pressure on the skin associated with steroid injection poses a risk of petechiae, micro-haemorrhagic spots, dense pigmentation, atrophy, and even ulceration. This was a handicap in one of the earlier patients treated. After some consideration it was decided to use Vitamin $\mathrm{E}$ on the skin surface before placing the moisture-retaining dressing. Vitamin $\mathrm{E}$ is a highly potent free radical scavenger and an antioxidant $[27,28]$. Vitamin $\mathrm{E}$ is also known to be a cell membrane lipid stabilizer, which protects cell membrane from lipid peroxidation $[27,29]$. Since addition of Vitamin $E$ to the protocol there have been no episodes of acute skin problems, thus it appears that topical application of Vitamin E protects from pressure-induced damage to the skin.

\subsection{Materials and Methods (Tripoli Protocol)}

Between August 1996 and July 2005, 60 patients with 79 keloids were accrued from Tripoli Medical Centre in Tripoli, Libya and the Princess Norah Oncology Centre in Jeddah, Saudi Arabia. Age of this group of patients is between six and 70 (one patient) years. The majority of patients were between 21 and 30 years. The male to female ratio was 1:2. From this group 71 lesions in 57 patients could be accepted as per our criteria for inclusion. Remaining lesions were found not suitable mainly due to the anatomical site of the lesion where pressure could not effectively be applied i.e., anterior abdominal wall, the neck region, or because of the large size of the lesions itself.

\section{Patient Selection}

There were a huge number of referrals for treatment in this protocol. We set the inclusion and exclusion criteria as follows.

\subsection{Criteria for Eligibility}

- Patients who had recurrence after surgery and/or radiotherapy.

- Patients who could not be treated primarily by radiotherapy due to anatomical site of the lesions, i.e., breast, anterior neck, abdomen, etc.

- Reasonable volume and size of the lesion, which can be effectively infiltrated by drugs as prescribed $(6 \times 6$ $\times 2 \mathrm{~cm}$ average volume).

- Young children and adolescents with only symptommatic keloids, where radiation is not recommended.

\subsection{Criteria for Ineligibility}

- Patients with lesions where effective pressure cannot be applied; i.e., anterior abdominal wall, anterior neck etc. 
- Steroid allergy or known intolerance to steroids.

- Skin condition, which precludes pressure application namely, Psoriasis or Dermatitis close to the lesion.

- Patients with known coagulopathy.

\subsection{Criteria for Exclusion}

- Intolerance to regimen at any time during the course of the treatment.

- Physical or symptomatic deterioration at any stage during the course of treatment.

One common site of presentation was presternal lesions. Presternal keloids are known to be exceptionally resistant to therapy. Other common sites were the shoulder region and chest wall. Table 1 shows a list of involvement of various anatomical sites at presentation. Intractable irritation, cosmetic concern, and pain are most frequent presenting symptoms (Table 2). Frequently, patients were referred after having had multiple surgeries and other modalities of therapy. Less than a third of the group presented without having any therapy for keloid. Table 3 lists details of treatments received by patients prior to starting on this protocol.

\section{Techniques}

Once the patient (Figure 1) is accepted for treatment a careful mapping of the lesion is carried out (Figure 1(a)). A wax block between 1 and $2 \mathrm{~cm}$ thick is made to fit the

Table 1. Various anatomical sites of presentation of keloids in this series, in order of frequency.

\begin{tabular}{ccc}
\hline Anatomic site & No. of Keloids & \% \\
\hline Presternal & 16 & $20.2 \%$ \\
Shoulder & 10 & $12.6 \%$ \\
Chest wall & 18 & $22.7 \%$ \\
Neck (nape) & 3 & $3.8 \%$ \\
Arm and wrist & 5 & $6.3 \%$ \\
Trunk & 11 & $14.0 \%$ \\
Supra pubic & 2 & $2.5 \%$ \\
Submental & 2 & $2.5 \%$ \\
Thigh & 2 & $2.5 \%$ \\
Multiple post burn & 5 & $6.3 \%$ \\
Gluteal & 1 & $1.2 \%$ \\
Breast & 3 & $3.7 \%$ \\
Axilla (lateral chest wall) & 1 & $1.2 \%$ \\
TOTAL & $\mathbf{7 9}$ & $\mathbf{1 0 0 \%}$ \\
\hline
\end{tabular}

Table 2. Frequencies of various symptoms of patients in this study presented initially.

\begin{tabular}{ccc}
\hline Symptoms & No. of keloids & \% \\
\hline Irritation & 49 & $69 \%$ \\
Poor cosmetic reason & 42 & $59 \%$ \\
Pain & 33 & $46.4 \%$ \\
Difficulty in keeping clothes on & 27 & $38.0 \%$ \\
Functional restriction & 9 & $12.6 \%$ \\
Mass & 15 & $21.1 \%$ \\
Discharge & 1 & $1.4 \%$ \\
TOTAL & & $\mathbf{1 0 0 \%}$ \\
\hline
\end{tabular}

Table 3. Various treatments this group of patients underwent before being referred to us for treatment for lesions which were suitable for treatment.

\begin{tabular}{cc}
\hline Type of treatment & No. of keloids \\
\hline Multiple surgeries without radiotherapy & 19 \\
Recurrence following single surgical excision & 18 \\
Recurrence postoperative RT & 7 \\
No prior therapy & 27 \\
TOTAL & $\mathbf{7 1}$ \\
\hline
\end{tabular}

mapped area (Figure 1(b)). Triemcinolone diacetate; (kenacort; laderele lab $40 \mathrm{mg} / \mathrm{ml}$ ) Dexamethasone (ampoules of $4 \mathrm{mg} / \mathrm{ml}$ : Hikma Pharmaceuticals. Amman, Jordan) and heparin (Hikma-Heparin $5000 \mathrm{iu} / \mathrm{ml}$ is mixed at 1:1:1 ratio). For 5 - 6 minutes these agents are mixed in the syringe by vigorous shaking. If the injection is painful (which frequently is the case), then $2 \%$ Xylocaine is infiltrated at the injection site. If a dermajet (PenteFerte $1 \mathrm{~mm} 25 \mathrm{~g} 0.5 \times 16 \mathrm{~mm}$, made in Italy) is not available, the lesion is firmly held between the left thumb and index, then infiltration is carried out to obtain true intradermal instillation (by inserting the needle horizontally to the surface of the skin). After installation, gentle massage is applied for 5 - 6 minutes to help dispersal of drugs into the intrices of the lesion. A thin layer of vita$\min \mathrm{E}$ is spread on the lesion, which is then covered by "Para film”. (Para film "M" Mtg. By American National Can TM. Greenwich CT. 06836, USA), which is made airtight by securing the Para film with "Tegaderm" (CE0086 3M Health Care, St. Paul MN 55144-1000 USA). Instead of using silicone sheets, Para film was opted for, which is as efficient at retaining moisture and making the lesion softer, but at a significantly lower cost. Other investigators also successfully tried with other non silicone- 


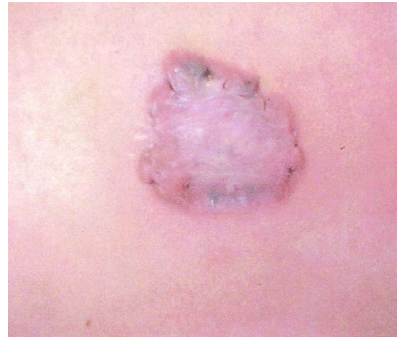

(a)

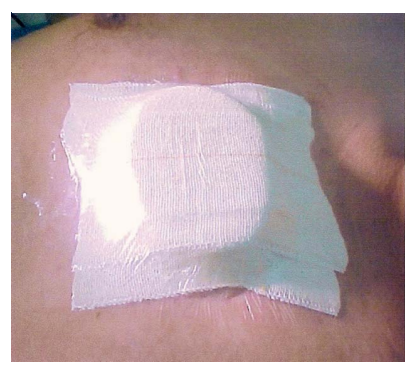

(c)

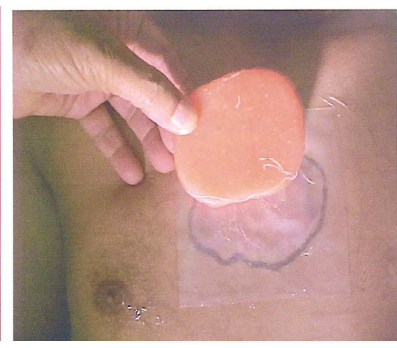

(b)

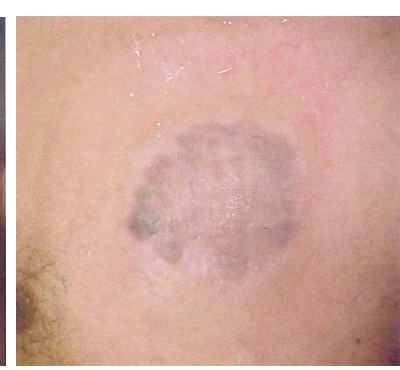

(d)
Figure 1. Demonstrating the steps of the procedure of concurrent multi-modality treatment of keloid. (a) Presternal butterfly keloid before treatment; (b) Following injection occlusive dressing with polyfilm and Tagaderm. The wax block to be used for compression is also seen; (c) Wax blocks secured on the lesion above the occlusive dressing, compressed with Elastoplast; (d) Complete flattening of the lesion with residual skin pigmentation. 3 months after completion of 3 injections.

based materials [30]. "Para film" is readily available in most clinical laboratories. The wax mould is placed on the lesion to match the outline. The wax moulding is secured and pressure dressing applied with elastoplasts (Figure 1(c)). Patients are reviewed weekly and injected after 3 weeks. Three injections are normally planned. However, application of pressure continues, at times, for 24 - 30 weeks to obtain maximal flattening.

\section{Results}

It has been difficult to quantitate and measure the response of therapy in this situation; therefore, an objective classification system of response of keloids to treatment was devised. Measured regression of the lesion, documented symptomatic improvement, and also index of satisfaction were collected for analysis. Results of which are as follows.

\section{Definition of Response}

Complete Response: Unlike many other clinical situations, the texture of the skin surface cannot be changed. Therefore, in this group, it is considered a complete response when:
- The thickness of the lesion is less than $25 \%$ of its presenting height or completely flattened;

- All presenting symptoms have disappeared;

- Patient is "satisfied" with the cosmetic result (optional).

Partial Response:

- When thickness of the lesion remains $25 \%$ - $75 \%$ of its original presentation;

- Persistence of any presenting symptoms or all presenting symptoms persist with less intensity;

- Cosmetic result is at least "acceptable" as opposed to "satisfactory" (optional).

No response or progression:

- When there is no change in the thickness of the lesion ( $0 \%$ - $25 \%$ reduction);

- No change or exacerbation of presenting symptoms;

- Both of the above.

\section{Index of Satisfaction}

The majority of the patients presented with multiple symptoms and had undergone repeated treatment without any benefit except deteriorating cosmesis. We asked all patients (or parents) about their level of satisfaction following completion of treatment. They had to respond with a " $\sqrt{ }$ " in an unmarked questionnaire whether the post treatment appearance was "satisfactory", "acceptable", or "no comment".

\section{Response to Treatment}

The result of the treatment is presented in Table 4. We made every endeavor to assess the response to treatment objectively by using the above criteria and by assessing patients' index of satisfaction in this group of 71 patients, who received treatment with this technique (CMTK).

\section{Results}

The tolerance and compliance to treatment was acceptable:

As per our criteria complete response was achieved in 55 (77\%) lesions, 13 (19\%) lesions had some relief of symptoms and three (3) had no response (4\%) (Table 4).

Cosmetic result was "satisfactory" amongst 36 lesions (50\%), "acceptable" amongst another 21 lesions (44\%) and "no comment" was expressed regarding four (4) keloids (6\%) (Table 4).

Lesions in the limb, gluteal region, and neck presented with painful restriction of movement. Twelve (12) keloids presented with functional restriction. Following completion of therapy, full functional recovery was achieved in three (3) out of 12 lesions. Partial functional recovery was achieved in the remaining nine (9). It is 
Table 4. Gives details of response to treatment using CMTK Protocol (Tripoli Protocol). 71 keloidal lesions were treated according to the Protocol.

\begin{tabular}{ccc}
\hline Parameter & $\begin{array}{c}\text { No. of } \\
\text { keloids }\end{array}$ & \% \\
\hline Complete response & 55 & $77 \%$ \\
Partial response & 13 & $19 \%$ \\
No response & 3 & $4 \%$ \\
Cosmesis “Satisfactory” per patient & 36 & $50 \%$ \\
Cosmesis "Acceptable” & 31 & $44 \%$ \\
Cosmesis “Unacceptable” & 4 & $6 \%$ \\
Pre-treatment functional impairment & $12 / 71$ & $16.6 \%$ \\
Post therapy full recover & $3 / 12$ & $25 \%$ \\
Post therapy partial functional recovery & $9 / 12$ & $75 \%$ \\
\hline
\end{tabular}

interesting to note that all lesions had at least some degree of functional recovery.

Figure 1(a) shows a 28-year-old male infantry soldier, who developed a keloid in the presternal region on an acne site, which had developed to the presenting size over a period of $3-4$ years. Six months previously he started to have severe itching, pain, and discomfort while wearing clothes. He had, on two occasions, intra-lesional triamcinolone injections, with only a temporary period of relief. The lesion increased slightly in size with clinical diagnosis of keloid. He was started on the concurrent multimodality regimen; Figure 1(b) shows the mapping of the lesion, covered by polyfilm and Tegaderm occlusive (airtight) dressing. This also demonstrates the customized wax block to be placed on the mapped area of the lesion to exert continuous pressure (compressive dressing). Figure 1(c) shows the wax block in place and pressure applied by Elastoplast, which is secured by a second layer of Tegaderm. Figure 1(d) shows the lesion after six (6) months of treatment. This is after four (4) applications of concurrent multimodality (CMTK) regimen; the lesion is almost flat, slightly pigmented, and totally asymptomatic.

Figure 2(a) shows post-burn keloids in the scapular ${ }^{\circledR}$ region in a 38-year-old male Filipino patient. Figure 2(b) after 2 episodes of multimodality therapy. The lesion has been completely flattened, normal skin covering, and excellent cosmesis

Figure 3(a) shows recurrence of a left deltoid region keloid following primary resection of a vaccination keloid in a 35-year-old Saudi male. Figure 3(n) shows the lesion completely flat with some pigmentation and asymptomatic 3 months after completion of 3 episodes of multimodality treatment (CMTK).

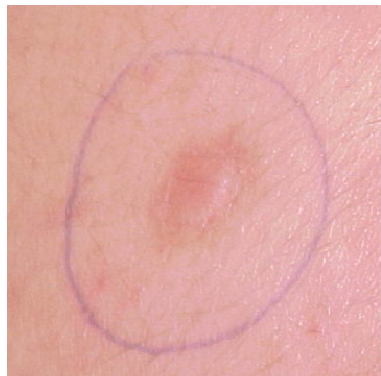

(a)

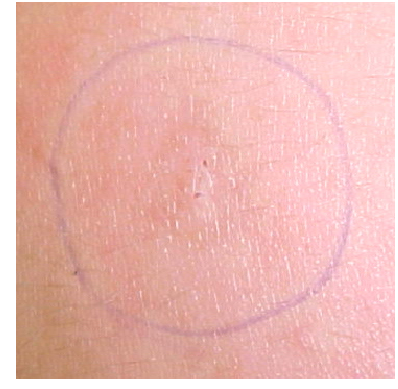

(b)
Figure 2. Post burn keloid. (a) Post burn keloid in the shoulder (left), before treatment; (b) Complete resolution following the first treatment.

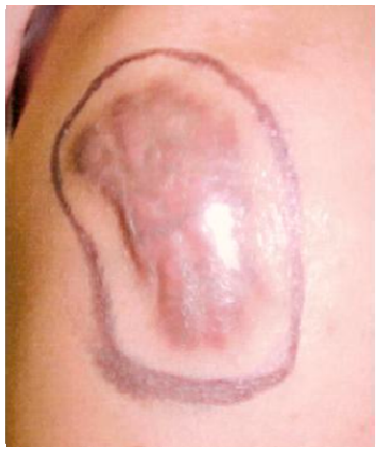

(a)

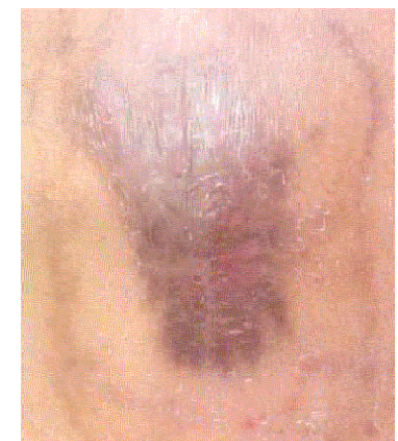

(b)
Figure 3. (a) The lesion before treatment; (b) Complete flattening of the lesion after treatment with residual pigmentation.

Figure 4(a) post-sternotomy severely symptomatic keloid in an 18-year-old female. Figure 4(b) shows complete regression and symptomatic relief of the keloid after 3 treatments.

Figure 5(a) shows multiple post varicella keloids in a 19-year-old Saudi girl. She presented with 15 lesions, all were treated in stages and all responded completely with some residual pigmentation (Figure 5(b)).

Tolerance to the concurrent multimodality (CMTK) regimen has been excellent. There were minor side effects, namely pigmentation, skin atrophy, etc., which have been documented in Table 5.

\section{Discussion}

Keloids are not life threatening and, in a way, self-limiting. Dismal results of treatment of recurrent, large, and bulky lesions have so far alienated the medical community. The underlying mechanism of keloid formation is unclear. Significant strides have been made to understand the molecular mechanism [2]. As a result, post-operative recurrence can now be effectively eliminated, but a cure for matured unresectable keloids still remains elusive. The result of postoperative external beam radiotherapy 


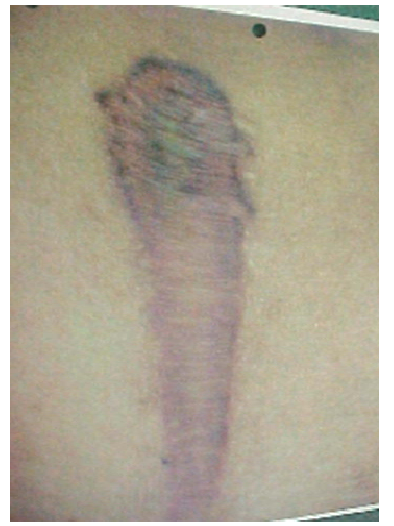

(a)

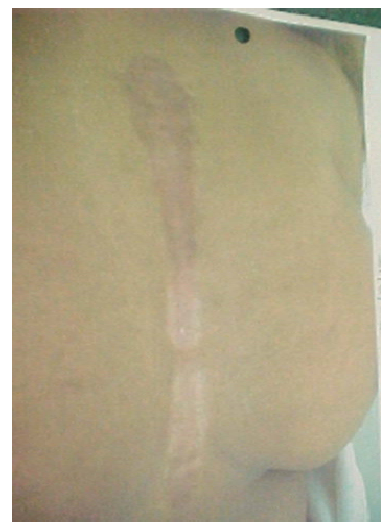

(b)
Figure 4. Post sternotomy keloid. (a) Before treatment; (b) Six months after treatment complete regression with residual depigmentation seen.

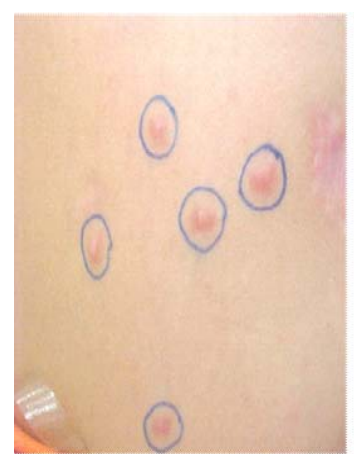

(a)

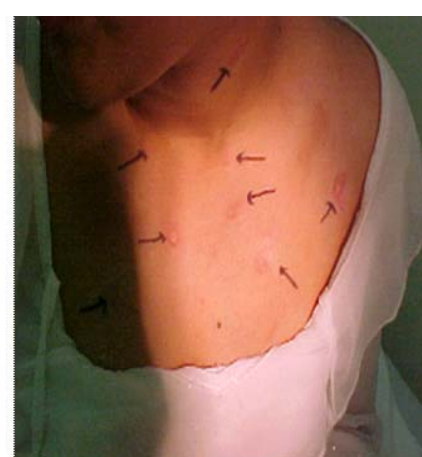

(b)
Figure 5. Multiple keloids following chicken pox. (a) Several keloids in the chest wall before treatment (see text), patient; (b) Complete regressions of all the lesions with faint residual pigmentation, 6 months after completion of scheduled treatment.

Table 5. Indicated complication and side efects of CMTK regimen.

\begin{tabular}{rc}
\hline Complication & No. of Lesions \\
\hline Pigmentation & 28 \\
Skin trophy & 3 \\
Wound dehiscence & 0 \\
Steroid induced toxicity & 0 \\
Wound healing problem & 0 \\
No response & 3 \\
Petechiae & 2 \\
Bleeding/Haematoma & 0
\end{tabular}

[31] or brachytherapy $[16,24]$ is excellent. On the other hand, primary radiotherapy for treatment of keloids until recently proved to be essentially ineffective. Malaker, et $a l$. demonstrated a hypo-fractionated radiation therapy regimen treated successfully as primary treatment for unresectable keloids. They used once weekly 750 cGy on Kilovoltage or $3-6 \mathrm{MeV}$ electrons to treat primarily with radiation, to a total dose of 3750 cGy in five weeks. Epilation was the most common acute and late toxicity. Tolerance and compliance were excellent [32]. In an attempt to get the greatest benefit, the agents known to be effective have been used in this protocol in a concerted fashion. Three agents, namely long acting steroids, occlusive dressing, and pressure, are all known to have beneficial effect against keloids. The addition of Heparin may have enhanced the effect of steroids to reduce neovascularity and induce regression. Unlike malignant tumours, in keloids, angiogenicity is not an issue. It is known that hypoxia is a strong trigger for neo-angiogenesis [33-35]. Chronic hypoxia is also known to cause fibroblastic proliferation [1,25,36,37]. By applying pressure to a keloidal lesion, it is possible to initiate exacerbation of keloidal growth. On the other hand, if the mechanical pressure is high enough, it is possible to occlude keloidal vessels to such an extent that nutrition and oxygen supply will be restricted sufficiently to induce atrophy. The amount of pressure needed to achieve this effect, may also cause atrophy or even necrosis of the superficial layer of the overlying skin. In fact, the first patient in this group did have significant petechiae, desquamation, and minute focal ulceration after the first application of pressure. Therefore, we opted for less pressure and topical vitamin E application on the skin to prevent atrophy and/or necrosis. This appears to have worked for this cohort. By inducing hypoxia, if angiogenesis is provoked, it would further enhance the growth of the lesion. In fact, it was noted that if only pressure is applied for these lesions for a week then removed, not immediately but after 5 - 6 days, the lesion increases in size, becomes hyperemic, tender, and appears more active. This led us to postulate that pressure induced hypoxia may have provoked neo-angiogenesis in the lesion, which is why they became active 5 - 6 days after releasing the pressure. Under light microscopy keloidal tissues have much less vascularity than normal skin, but under electron microscopy Kischer, et al. [22,23] noticed rich micro-vascularity in the keloidal tissue. Significant numbers of these vessels remained completely or partly obliterated and had dense walls. It is possible that added hypoxia due to pressure induces further production of vascular endothelial growth factors (VEGF) and thus neo-angiogenesis and further proliferation of microvasculature and keloidal regrowth to be clinically evident as we noticed in some of our patients when application of pressure was abandoned after 1 or 2 treatments. 
It is through the combined anti-angiogenic effect of long acting steroid and heparin infiltration in the lesion that should be able to counteract pressure-induced neoangiogenesis, at least during the early phase of treatment when it is most needed. It appears that this hypothesis may be true in our patients who achieved an excellent regression.

Silicone sheet and silicon gel seems to produce symptomatic relief and also softening of the keloidal lesion, by moisture retention [12-14]. This is likely to loosen the tightly packed collagen bundles in the keloidal lesion and improve oxygenation and nutrition by diffusion. This may also cause diffusion of symptoms causing agents out of the lesion resulting in symptomatic relief [21,38]. Silicone sheets and silicone gel are expensive and are not readily available. Therefore, we opted to use "Para film" which is commonly used in all clinical laboratories, and is cheap and easily available. This has the similar moisture-retaining property as the "Silicone products".

To increase durability of Parafilm we have used 3 - 4 folds of the material. To ensure air-tightness of the dressing we used Tegaderm, but any type of tape (non allergic) can be as effective as Tegaderm. It appears that several factors are working at cross purposes in this protocol. However, the net outcome is more favorable than one could have envisaged. By avoiding use of ionizing radiation, we have made this protocol applicable to children and women. There is no risk of carcinogenesis, growth impairment, or organ failure as known to occur in case of treatment associated with ionizing radiation.

Frequently in our geographical environment, distance of the clinic from the patient's hometown is a deterring factor for patients to come for regular follow-up. Fast progressing and universally available communication systems hopefully will bridge the gap not only in follow-up of this group of patients, but also for all patient populations in general. Late complications of atrophy, ulceration, recurrence, or malignant transformation have not been reported by any of our patients in the study.

Maintaining a regular follow-up for these patients has been more difficult because otherwise physically well and active patients are generally non-compliant for follow-ups [31,38-40]. Further trials with a larger patient population and long-term follow up would certainly give a proper perspective of this regime.

\section{Conclusion}

This is a simple, affordable, and universally applicable technique to offer some comfort to hitherto untreatable symptomatic keloids. The procedure is acceptable. Response rate is significant at $77 \%$, and patient compliance is also very good. Toxicities associated with the technique are minimal. Most importantly this can be safely used for children, unlike radiation. The follow-up has been from 6 - 60 months. Further studies with a larger population in the prevalent areas for keloid i.e. Sub-Saharan Africa and Southern Asia should be useful. With some perseverance one can master this technique easily. This technique can be applied in an outpatient clinic or for that matter in the office of a General Practitioner or a primary health care facility.

\section{Acknowledgements}

The authors wish to thank Sister Kim Geong Ye, Chief Radiotherapy Nurse at Tripoli Medical Centre, Sister Rene Cornellissen and Fuad Salameh, Staff Nurse of Radiotherapy Section of Princess Norah Oncology Centre for their meticulous care of our patients, and Mrs. Noura Pellicci, Princess Norah Oncology Centre secretariat for preparing the manuscript. Special thanks to Miss Joan Joseph and Mr. Irus Toussaint of Ross University School of Medicine for administrative and technical support.

\section{REFERENCES}

[1] S. Anderson and B. M. Branner, "Progressive Renal Disease: A Disorder of Adaptation," Quarterly Journal of Medicine, Vol. 70, No. 263, 1989, pp. 185-189.

[2] P. Rubin, A. Soni and J. P. Williams, "The Molecular and Cellular Biologic Basis for the Radiation Treatment of Benign Proliferative Disease," Seminars in Radiation Oncology, Vol. 9, No. 2, 1999, pp. 203-214. doi:10.1016/S1053-4296(99)80010-1

[3] A. E. Brissett and D. A. Sherris, "Scar Contractures, Hypertrophic Scars, and Keloids," Facial Plastic Surgery, Vol. 17, No. 4, 2001, pp. 263-272. doi:10.1055/s-2001-18827

[4] W. G. Payne, F. Ko, S. Anspaugh, et al., "Down-Regulating Causes of Fibrosis with Tamoxifen: A Possible Cellular/Molecular Approach to Treat Rhinophyma," Annals of Plastic Surgery, Vol. 56, No. 3, 2006, pp. 301305. doi:10.1097/01.sap.0000199155.73000.2f

[5] H. Tamai, O. Katoh, S. Suzuki, et al., "Impact of Tranilast on Restenosis after Coronary Angioplasty: Tranilast Restensosis Following Angioplasty Trial (TREAT)," American Heart Journal, Vol. 138, No. 5, 1999, pp. 968-975. doi:10.1016/S0002-8703(99)70025-6

[6] G. Muneuchi, S. Suzuki, M. Onodera, O. Ito, Y. Hata and H. H. Igawa, "Long-Term Outcome of Intralesional Injection of Triamcinolone Acetonide for the Treatment of Keloid Scars in Asian Patients," Scandinavian Journal of Plastic and Reconstructive Surgery and Hand Surgery, Vol. 40, No. 2, 2006, pp. 111-116. doi:10.1080/02844310500430003

[7] G. G. Gauglitz, H. C. Korting, T. Pavicic, et al., "Hypertrophic Scarring and Keloids: Pathomechanisms and Current and Emerging Treatment Strategies,” Molecular Medicine, Vol. 17, No. 1-2, 2011, pp. 113-125.

doi:10.2119/molmed.2009.00153 
[8] S. Mutalik, "Treatment of Keloids and Hypertrophic Scars," Indian Journal of Dermatology, Venereology and Leprology, Vol. 71, No. 1, 2005, pp. 3-8. doi:10.4103/0378-6323.13777

[9] A. Goel and P. Shrivastava, "Post-Burn Scars and Scar Contractures,” Indian Journal of Plastic Surgery, Vol. 43, 2010, pp. S63-S71. doi:10.4103/0970-0358.70724

[10] B. Berman and H. C. Bieley, "Keloids," Journal of the American Academy of Dermatology, Vol. 33, No. 1, 1995, pp. 117-123. doi:10.1016/0190-9622(95)90035-7

[11] T. S. Alster and E. L. Tanzi, "Hypertrophic Scars and Keloids: Etiology and Management," American Journal of Clinical Dermatology, Vol. 4, No. 4, 2003, pp. 235243. doi:10.2165/00128071-200304040-00003

[12] G. L. Dockery and R. Z. Nilson, "Treatment of Hypertrophic and Keloid Scars with Silastic Gel Sheeting," Journal of Foot and Ankle Surgery, Vol. 33, No. 2, 1994, pp. 110-119.

[13] K. Perkins, R. B. Davey and K. A. Wallis, "Silicone Gel: A New Treatment for Burn Scars and Contractures," Burns, Including Thermal Injury, Vol. 9, No. 3, 1983, pp. 201-204. doi:10.1016/0305-4179(83)90039-6

[14] M. Gibbons, R. Zuker, M. Brown, et al., "Experience with Silastic Gel Sheeting in Pediatric Scarring," Journal of Burn Care \& Rehabilitation, Vol. 15, No. 1, 1994, pp. 69-73. doi:10.1097/00004630-199401000-00013

[15] B. Hirshowitz, E. Lindenbaum, Y. Har-Shai, et al., "Static-Electric Field Induction by a Silicone Cushion for the Treatment of Hypertrophic and Keloid Scars," Plastic and Reconstructive Surgery, Vol. 101, No. 5, 1998, pp. 11731183.

[16] L. Narkwong and P. Thirakhupt, "Postoperative Radiotherapy with High Dose Rate Iridium 192 Mould for Prevention of Earlobe Keloids," Journal of the Medical Association of Thailand, Vol. 89, No. 4, 2006, pp. 428-433.

[17] E. Willich, H. Kuttig, G. Pfeil, et al., "Pathological Changes (Developmental/Growth Disturbances) of the Spine after Radiation Therapy of Nephroblastomas during Early Childhood. A Retrospective Long-Term Follow-Up Study in 82 Children," Strahlentherapie und Onkologie, Vol. 166, 1990, pp. 815-821.

[18] D. L. Preston, A. Mattsson, E. Holmberg, et al., "Radiation Effects on Breast Cancer Risk: A Pooled Analysis of Eight Cohorts,” Radiation Research, Vol. 158, No. 2, 2002, pp. 220-235.

doi:10.1667/0033-7587(2002)158[0220:REOBCR]2.0.C $\underline{\mathrm{O} ; 2}$

[19] F. Nguyen, C. Rubino, S. Guerin, et al., "Risk of a Second Malignant Neoplasm after Cancer in Childhood Treated with Radiotherapy: Correlation with the Integral Dose Restricted to the Irradiated Fields," International Journal of Radiation Oncology Biology Physics, Vol. 70, No. 3, 2008, pp. 908-915. doi:10.1016/j.ijrobp.2007.10.034

[20] T. A. Mustoe, "Evolution of Silicone Therapy and Mechanism of Action in Scar Management," Aesthetic Plastic Surgery, Vol. 32, No. 1, 2008, pp. 82-92. doi:10.1007/s00266-007-9030-9
[21] P. W. Grigsby, A. Russel, D. Bruner, et al., "Late Injury of Cancer Therapy on the Female Reproductive Tract," International Journal of Radiation Oncology Biology Physics, Vol. 31, No. 5, 1995, pp. 1281-1299. doi:10.1016/0360-3016(94)00426-L

[22] C. W. Kischer, M. R. Shetlar and M. Chvapil, "Hypertrophic Scars and Keloids: A Review and New Concept Concerning Their Origin,” Scanning Electron Microscopy, Vol. 4, 1982, pp. 1699-1713.

[23] D. Deveci, J. M. Marshall and S. Egginton, "Chronic Hypoxia Induces Prolonged Angiogenesis in Skeletal Muscles of Rat,” Experimental Physiology, Vol. 87, No. 3, 2002, pp. 287-291. doi:10.1113/eph8702377

[24] B. Liu and M. K. Connolly, "The Pathogenesis of Cutaneous Fibrosis," Seminars in Cutaneous Medicine and Surgery, Vol. 17, No. 1, 1998, pp. 3-11. doi:10.1016/S1085-5629(98)80055-2

[25] C. T. Taylor and S. P. Colgan, "Therapeutic Targets for Hypoxia-Elicited Pathways,” Pharmaceutical Research, Vol. 16, No. 10, 1999, pp. 1498-1505. doi:10.1023/A:1011936016833

[26] P. Martin, "Wound Healing-Aiming for Perfect Skin Regeneration," Science, Vol. 276, No. 5309, 1997, pp. 75-81. doi:10.1126/science.276.5309.75

[27] M. G. Traber and L. Packer, "Vitamin E: Beyond Antioxidant Function," The American Journal of Clinical Nutrition, Vol. 62, No. 6, 1995, pp. 1501S-1509S.

[28] S. Khanna, S. Roy, H. Ryu, P. Bahadduri, P. W. Swaan, R. R. Ratan and C. K. Sen, "Molecular Basis of Vitamin E Action. Tocotrienol Modulates 12-Lipoxygenase, a Key Mediator of Glutamate-Induced Neurodegeneration,” The Journal of Biological Chemistry, Vol. 278, No. 44, 2003, pp. 43508-43515. doi:10.1074/jbc.M307075200

[29] L. Ernster, P. Forsmark and K. Nordenbrand, "The Mode of Action of Lipid-Soluble Antioxidants in Biological Membranes: Relationship between the Effects of Ubiquinol and Vitamin E as Inhibitors of Lipid Peroxidation in Submitochondrial Particles," Biofactors, Vol. 3, No. 4, 1992, pp. 241-248.

[30] V. Jones, J. E. Grey and K. G. Harding, “ABC of Wound Healing: Wound Dressings,” British Medical Journal, Vol. 332, No. 7544, 2006, pp. 777-780. doi:10.1136/bmj.332.7544.777

[31] K. Malaker, K. Vijayraghavan, I. Hodson and T. Al Yafi, "Retrospective Analysis of Treatment of Unresectable Keloids with Primary Radiation over 25 Years," Clinical Oncology (Royal College of Radiologists), Vol. 16, No. 4, 2004, pp. 290-298. doi:10.1016/j.clon.2004.03.005

[32] T. S. Alster and T. B. West, "Treatment of Scars: A Review," Annals of Plastic Surgery, Vol. 39, No. 4, 1997, pp. 418-432. doi:10.1097/00000637-199710000-00014

[33] B. J. Moeller, Y. Cao, Z. Vujaskovic, C. Y. Li, Z. A. Haroon and M. W. Dewhirst, "The Relationship between Hypoxia and Angiogenesis," Seminars in Radiation Oncology, Vol. 14, No. 3, 2004, pp. 215-221. doi:10.1016/j.semradonc.2004.04.005

[34] C. W. Pugh and P. J. Ratcliffe, "Regulation of Angio- 
genesis by Hypoxia: Role of the HIF System,” Nature Medicine, Vol. 9, No. 6, 2003, pp. 677-684. doi:10.1038/nm0603-677

[35] J. M. Gleadle, B. L. Ebert, J. D. Firth and P. J. Ratcliffe, "Regulation of Angiogenic Growth Factor Expression by Hypoxia, Transition Metals, and Chelating Agents," American Journal of Physiology, Vol. 268, No. 6, 1995, pp. C1362-C1368.

[36] J. T. Norman, I. M. Clark and P. L. Garcia, "Hypoxia Promotes Fibrogenesis in Human Renal Fibroblasts," Kidney International, Vol. 58, No. 6, 2000, pp. 23512366. doi:10.1046/j.1523-1755.2000.00419.x

[37] Y. Ganat, S. Soni, M. Chacon, M. L. Schwartz and F. M. Vaccarino, "Chronic Hypoxia Up-Regulates Fibroblast Growth Factor Ligands in the Perinatal Brain and Induces Fibroblast Growth Factor-Responsive Radial Glial Cells in the Sub-Ependymal Zone,” Neuroscience, Vol. 112, No. 4, 2002, pp. 977-991. doi:10.1016/S0306-4522(02)00060-X

[38] A. P. Kelly, "Medical and Surgical Therapies for Keloids," Dermatology and Therapy, Vol. 17, No. 2, 2004, pp. 212-218. doi:10.1111/j.1396-0296.2004.04022.x

[39] Q. Dinh, M. Veness and S. Richards, "Role of Adjuvant Radiotherapy in Recurrent Earlobe Keloids," Australasian Journal of Dermatology, Vol. 45, No. 3, 2004, pp. 162-166. doi:10.1111/j.1440-0960.2004.00079.x

[40] E. E. Tredget, B. Nedelec, P. G. Scott and A. Ghahary, "Hypertrophic Scars, Keloids, and Contractures. The Cellular and Molecular Basis for Therapy,” Surgical Clinics of North America, Vol. 77, No. 3, 1997, pp. 701-730. doi:10.1016/S0039-6109(05)70576-4 